\title{
Propagation des faisceaux gaussiens. Transport des faisceaux de puissance
}

\author{
A. Culoma
}

Laserdot, Route de Nozay, F-91460 Marcoussis, France

\section{Introduction}

Les caractéristiques optiques d'un faisceau laser sont très différentes de celles des faisceaux lumineux conventionnels issus de sources naturelles ou artificielles (soleil, lampes à incandescence, diodes électroluminescentes,....).

La description des transformations spatiales des faisceaux de lumière conventionnels fait appel aux théories de l'optique géométrique et de la radiométrie qui toutes deux s'accomodent de la notion de rayon lumineux. L'optique géométrique s'avère insuffisante pour décrire les transformations spatiales des faisceaux laser. La propagation des faisceaux laser fait largement appel au phénomène de diffraction. A cause de cela, l'intuition basée sur l'optique géométrique peut conduire à des résultats de propagation laser qui sont erronés.

\section{Faisceaux gaussiens}

\subsection{Intérêt de l'étude}

Par "faisceaux gaussiens", il faut comprendre non seulement le faisceau gaussien fondamental $\left(T E M_{00}\right)$ mais plus généralement les modes Hermito-gaussiens (TEM $M_{n m}$ ). Ces modes sont des modes propres de l'opérateur de propagation dans l'approximation paraxiale de l'équation d'onde scalaire et / ou de l'approximation de Fresnel de l'intégrale de diffraction de Huygens (qui sont deux approximations équivalentes).

Les modes Hermito-gaussiens constituent de plus une base "orthogonale" et "complète" des solutions de l'équation d'onde paraxiale sur laquelle tout faisceau laser arbitraire peut être décomposé. Ces modes sont connus également comme étant les modes propres des cavités de type "stable" ouverte et vide de milieu amplificateur dont le mode fondamental TEM $M_{00}$ est le plus répandu parmi les sources laser d'alignement. (laser Hélium-Néon)

\subsection{Propagation libre des faisceaux gaussiens}

L'équation d'onde scalaire dans son approximation paraxiale (valable jusqu'à des inclinaisons locales du front d'onde de $\pm 30^{\circ}$ par rapport à l'axe du faisceau) s'écrit :

$$
\frac{\partial u}{\partial z}=-\frac{j}{2 k}\left(\frac{\partial^{2} u}{\partial x^{2}}+\frac{\partial^{2} u}{\partial y^{2}}\right)
$$

avec

. z : axe privilégié de propagation

$. u(x, y, z)=E(x, y, z) e^{+j k z}$ où $E=$ champ électrique

$$
k=\frac{\omega}{c} n
$$

Une solution de cette équation est constituée par le mode TEMoo : (Fig. 1a)

$$
u(r, z)=A(z) \exp \left(-j k \frac{r^{2}}{2 q(z)}\right)
$$




$$
\text { avec } \quad \begin{aligned}
\frac{1}{q(z)} & =\frac{1}{R(z)}-j \frac{\lambda}{\pi \omega^{2}(z)} \\
\cdot q(z) & =\text { rayon complexe du faisceau } \\
\cdot R(z) & =\text { rayon de courbure du front d'onde } \\
\cdot \omega(z) & =\text { rayon en } \frac{1}{\theta^{2}} \text { du faisceau. }
\end{aligned}
$$

En injectant cette solution dans l'équation d'onde paraxiale, on obtient la loi de propagation du rayon complexe :

$$
\begin{aligned}
& q(z)=q(z=0)+z \\
& A(z)=A(z=0) \cdot \frac{q(z=0)}{q(z)}
\end{aligned}
$$

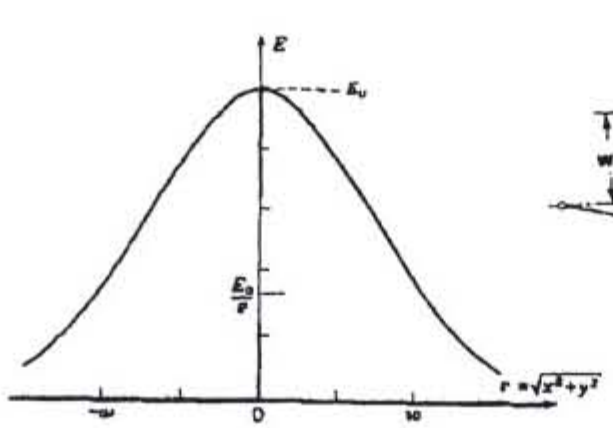

-Fig. 1.a-

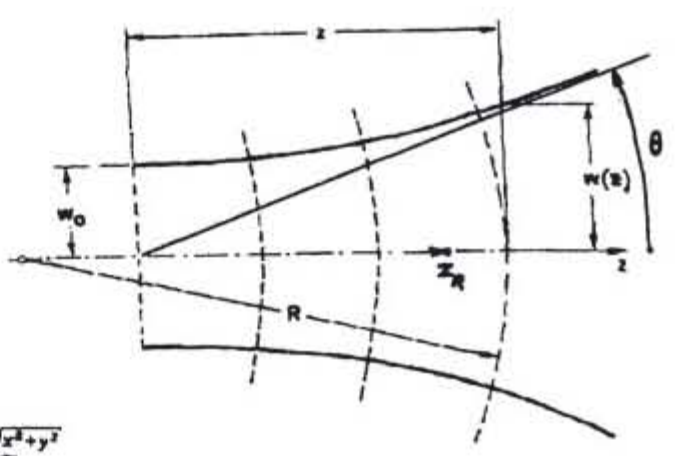

-Fig. 1.b-

A partir de (2) et (3), en remarquant qu'il existe un minimum pour w(z) noté wo qu'on place arbitrairement en $\mathbf{z}=0$ (par changement de coordonnées), on obtient par identification des parties réelles et imaginaires de (3), les relations d'évolution suivantes :

$$
\begin{aligned}
& \omega^{2}(z)=\omega_{0}^{2}\left(1+\left(\frac{z}{z_{R}}\right)^{2}\right) \\
& R(z)=z\left(1+\left(\frac{z_{A}}{z}\right)^{2}\right)
\end{aligned}
$$

$$
\text { avec } \quad z_{\mathrm{R}}=\frac{\pi \omega_{0}^{2}}{\lambda} \text { distance de Rayleigh }
$$

La relation $\omega(z)$ est représentée en Fig. 1b et décrit une hyperbole dont l'asymptote permet de définir la demi-divergence du faisceau $\theta_{1 / 2}=\frac{\lambda}{\pi \omega_{0}}$

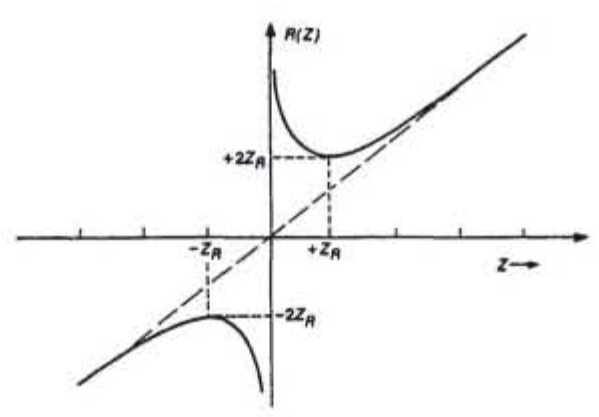


Propagation Libre (indice $n_{\text {. }}$ )

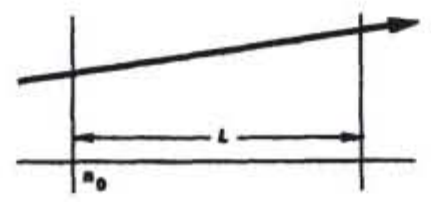

$$
\left[\begin{array}{cc}
1 & L / n_{0} \\
0 & 1
\end{array}\right]
$$

Lentille mince ( $f>0$ park une lentille convergente)

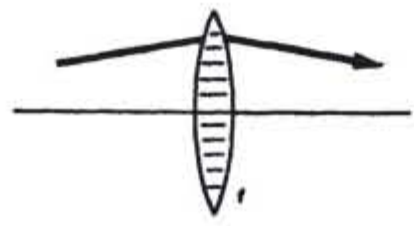

$$
\left[\begin{array}{cc}
1 & 0 \\
-1 / f & 1
\end{array}\right]
$$

Miroir sphärique, incidence $\theta \quad(R>0$ pour un miroir concave )

$$
R_{e}=R \cos \theta \text { dans le plare d'incidence }
$$

$R_{e}=R / \cos \theta$ dans el plan perpendiculaine au plan d'incidance

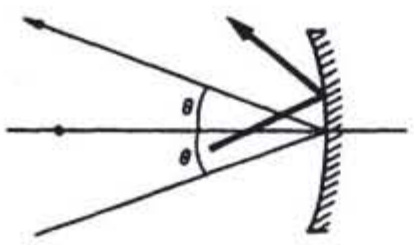

$\left[\begin{array}{cc}1 & 0 \\ -2 / R_{e} & 1\end{array}\right]$

kablean 1 
La relation $R(z)$ est représentée en Fig. 1c ; on peut noter que $|R(z)|$ présente un minimum pour l'abscisse correspondant à la distance de Rayleigh.

Les modes Hermito-gaussiens, solutions de l'équation d'onde paraxiale, sont dans le cas unidimensionnel de la forme :

avec

$$
u_{n}(x, z) \propto H_{n}\left(\frac{\sqrt{2} x}{\omega(z)}\right) \cdot \exp \left(-j k \frac{x^{2}}{2 R(z)}-\frac{x^{2}}{\omega^{2}(z)}\right)
$$

$$
\begin{aligned}
& \text {. } H_{n} \text { polynôme d'Hermite d'ordre } n \\
& H_{0}=1 \quad H_{1}(x)=2 x \text { et } \\
& H_{n+1}(x)=2 x H_{n}(x)-2 n H_{n-1}(x)
\end{aligned}
$$

. $R(z)$ et $\omega(z)$ sont exactement les mêmes paramètres que ceux développés

pour le faisceau fondamental TEMoo.

La figure 2 donne quelques représentations de taches laser correspondant aux modes TEM $_{n, m}$.
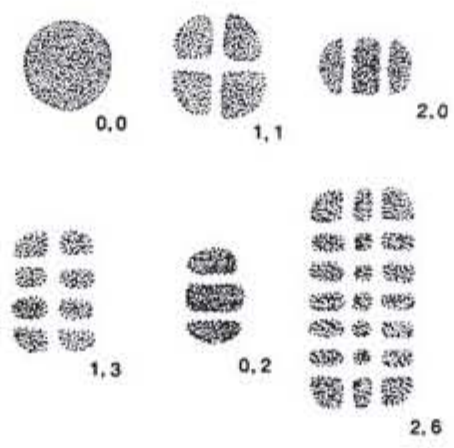

- Fig. 2 -

\subsection{Iransformation spatiale par un système optique paraxial.}

\subsubsection{Eormalisme matriciel de l'eptique géemétrique paraxiale.}

La transformation subie par un rayon lumineux se propageant entre deux plans encadrant un système optique paraxial peut être avantageusement décrite par une matrice agissant sur les coordonnées transverses et les cosinus directeurs d'un rayon issu du plan de départ.

(Fig. 3a)

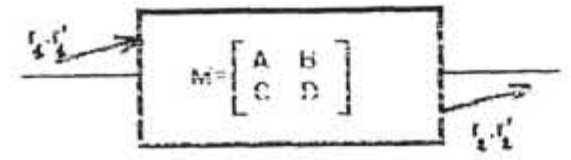

- Fig. 3.a -

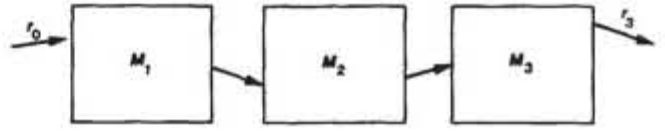

- Fig. 3.b -

Dans le cas unidimensionnel, la transformation peut s'écrire :

$$
\left(\begin{array}{l}
X_{2} \\
x_{2}^{\prime}
\end{array}\right)=\left(\begin{array}{ll}
A & B \\
C & D
\end{array}\right)\left(\begin{array}{l}
X_{1} \\
x_{1}^{\prime}
\end{array}\right)
$$

Quelques exemples de matrice de transformation sont données dans le tableau 1. 
Pour un système optique composé de diverses interfaces ou milieux mis en cascade, la matrice décrivant le système est simplement le produit de matrices des différents éléments constitutifs. (Fig. 3b)

$$
M=M_{3} M_{2} M_{1}
$$

\subsubsection{Eormule de transtormation soatiale des faisceaux gaussiens}

On peut montrer [1], qu'à partir des expressions matricielles exposées ci-dessus, il est possible de développer une relation du type "intégrale d'Huygens" reliant les amplitudes $u_{1}\left(x_{1}\right)$ et $u_{2}\left(x_{2}\right)$ se trouvant dans les plans d'entrée $\left(z_{1}\right)$ et de sortie $\left(z_{2}\right)$ du système optique paraxial décrit par la matrice $A B C D$ (Fig. 4)

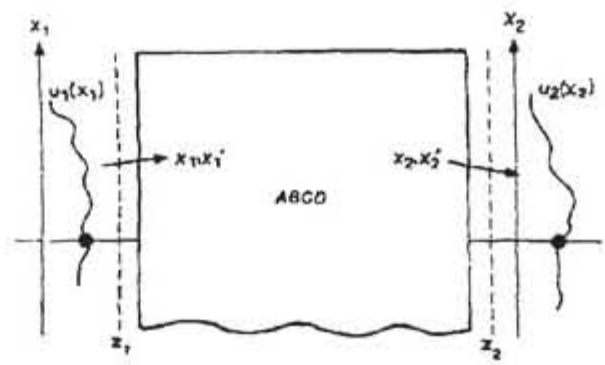

- Fig. 4 -

$u_{2}\left(x_{2}\right)=\sqrt{\frac{j}{B \lambda_{0}}} e^{-j k L_{0}} \int_{-\infty}^{+\infty} u_{1}\left(x_{1}\right) \exp \left(-j \frac{\pi}{B \lambda_{0}}\left(A x_{1}{ }^{2}-2 x_{1} x_{2}+D x_{2}^{2}\right)\right) d x_{1}$

où $L_{0}=$ chemin optique compté le long de l'axe optique entre les plans d'entrée $\left(z=z_{1}\right)$ et de sortie $\left(z=z_{2}\right)$.

$\lambda_{0}=$ longueur d'onde dans le vide.

Si on applique ce résultat au cas d'un faisceau gaussien où :

$$
u_{1}\left(x_{1}\right)=\exp \left(-j \frac{\pi x_{1}{ }^{2}}{q_{1} \lambda_{1}}\right) \quad \text { avec } \frac{1}{q_{1}}=\frac{1}{R_{1}}-j \frac{\lambda_{1}}{\pi \omega_{1}{ }^{2}}
$$

et en remarquant que $\int_{-\infty}^{+\infty} \exp \left(-a x^{2}-2 b x\right) d x=\sqrt{\frac{\pi}{a}} \exp \left(b^{2} / a\right)$

( $a$ et $b \in C$ et $R e a>0$ ) et $n_{1} \lambda_{1}=n_{2} \lambda_{2}=\lambda_{0}$, on obtient :

$$
\begin{aligned}
& u_{2}\left(x_{2}\right)=\sqrt{\frac{1}{A+n_{1} \frac{B}{q_{1}}}} \cdot \exp \left(-j \frac{\pi x_{2}^{2}}{q_{2} \lambda_{2}}\right) \\
& \operatorname{avec} \frac{q_{2}}{n_{2}}=\frac{A\left(q_{1} / n_{1}\right)+B}{C\left(q_{1} / n_{1}\right)+D}
\end{aligned}
$$


Cette relation (7) permet de décrire la propagation du faisceau gaussien à travers une séquence d'éléments optiques paraxiaux.

A titre d'exemple, on va établir les formules de conjugaison de "waist" d'un faisceau gaussien traversant une lentille mince (Fig. 5).

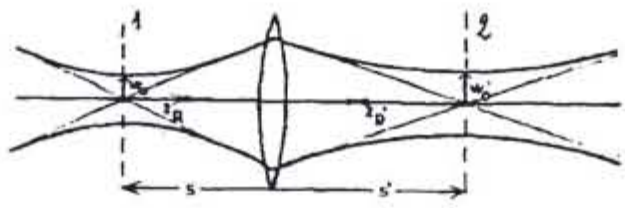

- Fig. 5 -

La matrice décrivant le passage du plan 1 au plan 2 s'écrit :

$$
\left(\begin{array}{ll}
1 & s^{\prime} \\
0 & 1
\end{array}\right)\left(\begin{array}{cc}
1 & 0 \\
-\frac{1}{f} & 1
\end{array}\right)\left(\begin{array}{ll}
1 & s \\
0 & 1
\end{array}\right)=\left(\begin{array}{cc}
1-\frac{s^{\prime}}{f} & s+s^{\prime}-\frac{s^{\prime}}{f} \\
-\frac{1}{f} & 1-\frac{s}{f}
\end{array}\right)=\left(\begin{array}{ll}
A & B \\
C & D
\end{array}\right)
$$

Si on suppose que le plan 1 est au waist "objet" alors $\frac{1}{q_{1}}=-j \frac{\lambda}{\pi \omega_{0}^{2}}$

de même, si le plan 2 est au waist "image" : $\frac{1}{q_{2}}=-j \frac{\lambda}{\pi \omega_{0}^{\prime}{ }^{2}}$

Si on applique la relation (7) entre les plans 1 et 2 , on obtient après identification des parties réelles et imaginaires :

$$
\left(\frac{s^{\prime}}{f}\right)=1+\frac{(s / f-1)}{(s / f-1)^{2}+\left(\frac{z R}{f}\right)^{2}}
$$

relation de conjugaison des positions de "waist" et

$$
m=\frac{\omega_{0}^{\prime}}{\omega_{0}}=\frac{1}{\left((1-s / f)^{2}+\left(z_{R} / f\right)^{2}\right)^{1 / 2}}
$$

relation de grandissement de "waist" et

$$
z_{R}^{\prime}=m^{2} z_{R}
$$

Les figures $6 a$ et $6 \mathrm{~b}$ représentent les relations (8) et (9) sous forme de famille de courbes paramétrées par le facteur $\left|z_{R} / f\right|$.

La convention de signe adoptée pour s et s' est la suivante :

$s>0$ si le waist objet est à gauche de la lentille mince

$\mathrm{s}^{\prime}>0$ si le waist image est à droite de la lentille mince. 

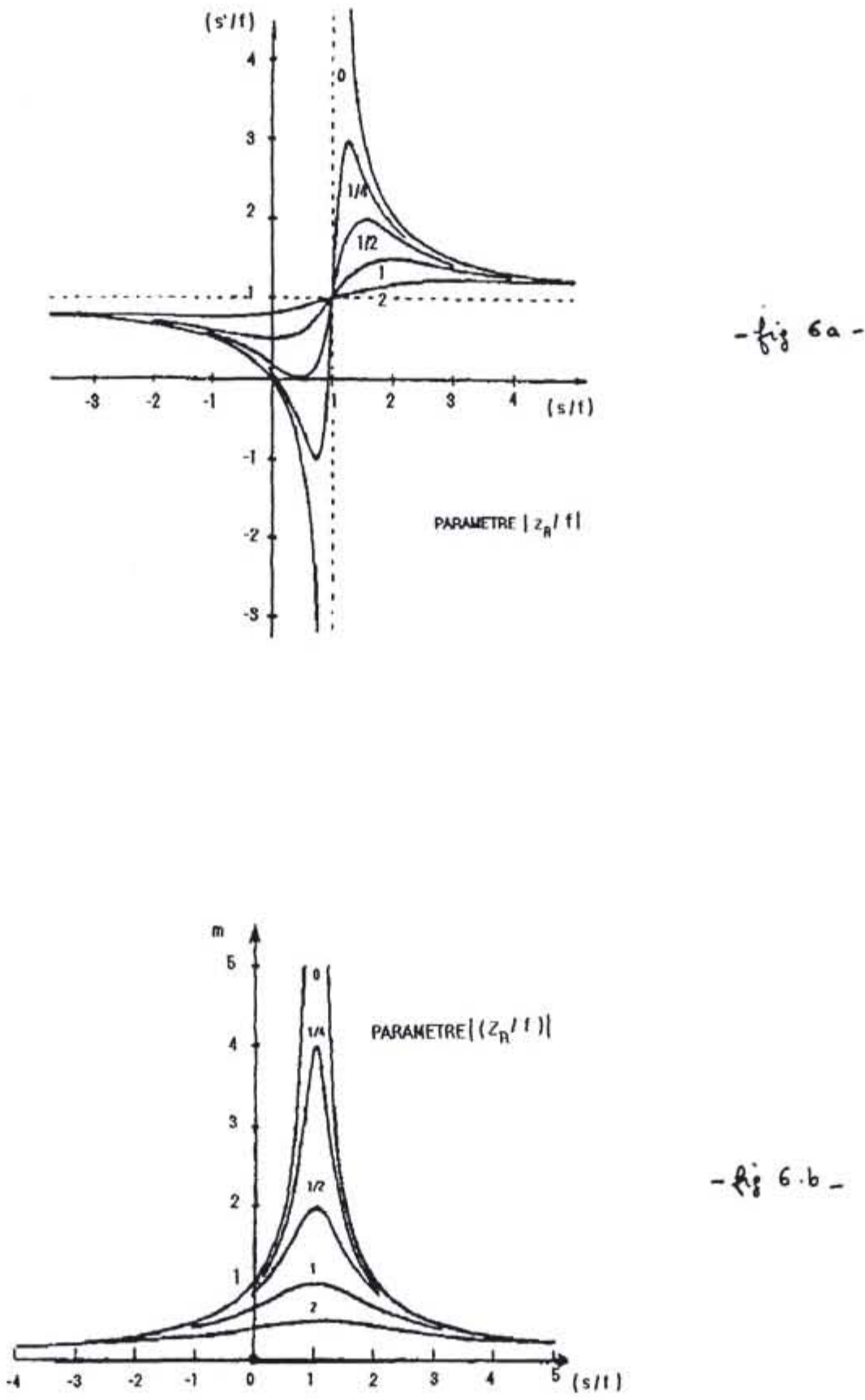
Remarque : Propagation des faisceaux gaussiens et Optique géométrique.

Considérons un diaphragme "fictif" placé en A (Fig. 7) et ayant un rayon |AB| égal au rayon en $1 / e^{2}, \omega(A)$ du faisceau incident; son image géométrique est située en $A^{\prime}$ et possède un rayon $\left|A^{\prime} B^{\prime}\right|$.

On peut montrer [2] que la faisceau gaussien transformé possède en $A^{\prime}$, un rayon $\omega^{\prime}\left(A^{\prime}\right)$ qui est égal à $\left|A^{\prime} B^{\prime}\right|$.

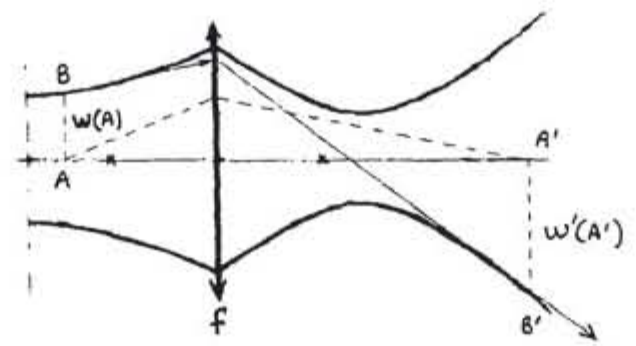

- Fig. 7 -

\subsection{Effet d'une troncature circulaire sur la propagation d'un faisceau gaussien}

Toutes les expressions analytiques de propagation établies jusqu'à présent supposent des dimensions de composants "suffisamment" grandes pour négliger les effets dûs à la diffraction par les bords du composant (ceci est vrai tant que le rayon du diaphragme circulaire "a" est supérieur à 2,1 $\omega_{1 / e^{2}}{ }^{2}$ ).

On peut montrer [3] que dans le cas d'une troncature légère $\left(a>1,1 \omega_{1 / e^{2}}\right)$, le faisceau en champ lointain peut être approximé par un faisceau gaussien ayant un waist fictif localisé au waist d'origine $\left(\omega_{0}, I_{0}\right)$ dont la dimension est $\omega^{\prime} 0$, l'intensité sur l'axe l'o et la divergence $\theta_{0}^{\prime} 0$ (Fig. 8) tels que :
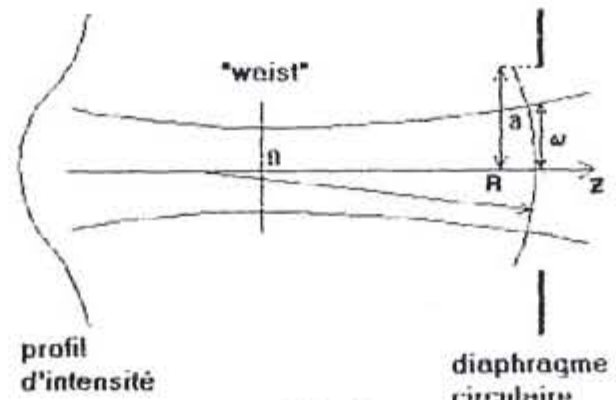

- Fig. 8 -

$$
\begin{aligned}
& \frac{\omega_{0}^{\prime}}{\omega_{0}} \sim 1-\sqrt{\Delta \mathrm{P} / \mathrm{P}} \cos \left(\pi \mathrm{a}^{2} / \mathrm{R}\right) \\
& \frac{\mathrm{I}_{0}}{\mathrm{I}_{0}} \sim\left(1-2 \sqrt{\Delta \mathrm{P} / \mathrm{P}} \cos \left(\pi \mathrm{a}^{2} / \lambda \mathrm{R}\right)\right)^{-1}
\end{aligned}
$$




$$
\begin{aligned}
& \frac{\theta^{\prime} 0}{\theta_{0}} \sim\left(1-\sqrt{\Delta \mathrm{P} / \mathrm{P}} \cos \left(\pi \mathrm{a}^{2} / \lambda \mathrm{R}\right)\right)^{-1} \\
& \text { avec } \frac{\Delta \mathrm{P}}{\mathrm{P}}=\exp \left(-2 \mathrm{a}^{2} / \omega^{2}\right)=\text { perte relative de puissance à la traversée de l'ouverture } \\
& \text { de rayon a } \\
& \mathrm{R}=\text { rayon de courbure du faisceau incident au niveau de l'ouverture diffractante. }
\end{aligned}
$$

Dans le cas de troncature sévère $\left(a<1,1 \omega_{1 / / e^{2}}\right)$, le faisceau n'est plus gaussien et les formules ci-dessus ne sont plus valables.

\subsection{Synthèse d'un système optique.}

II s'agit de trouver un système optique réalisant une transformation donnée sur un faisceau gaussien. Ceci revient à imposer une matrice en ABCD. Dans le cas où les milieux extrêmes sont de même indice, on montre [4] qu'une matrice ABCD quelconque peut s'exprimer sous la forme de produit de matrices "primitives" de la forme

En effet :

$$
\alpha=\left(\begin{array}{ll}
1 & \alpha \\
0 & 1
\end{array}\right) \text { et } \beta=\left(\begin{array}{ll}
1 & 0 \\
\beta & 1
\end{array}\right)
$$

$$
\begin{aligned}
& \begin{array}{l}
* \operatorname{sic} \neq 0 \\
-\operatorname{si} C=0
\end{array} \quad\left(\begin{array}{ll}
A & B \\
C & D
\end{array}\right)=\left(\begin{array}{cc}
1 & (A-1) / C \\
0 & 1
\end{array}\right)\left(\begin{array}{ll}
1 & 0 \\
C & 1
\end{array}\right)\left(\begin{array}{cc}
1 & (D-1) / C \\
0 & 1
\end{array}\right) \\
& B \neq 0 \quad\left(\begin{array}{ll}
A & B \\
C & D
\end{array}\right)=\left(\begin{array}{cc}
1 & 0 \\
(D-1) / B & 1
\end{array}\right)\left(\begin{array}{ll}
1 & B \\
0 & 1
\end{array}\right)\left(\begin{array}{cc}
1 & 0 \\
(A-1) / B & 1
\end{array}\right) \\
& \text { - si } \mathrm{B}=\mathrm{C}=0 \\
& \text { on se ramène aux } 2 \text { cas précédents en remarquant que : } \\
& \begin{aligned}
\left(\begin{array}{ll}
A & 0 \\
0 & D
\end{array}\right)=\left(\begin{array}{cc}
1 & \alpha \\
0 & 1
\end{array}\right)\left(\begin{array}{cc}
A & -\alpha D \\
0 & D
\end{array}\right) \\
=\left(\begin{array}{cc}
A & -\alpha A \\
0 & D
\end{array}\right)\left(\begin{array}{cc}
1 & \alpha \\
0 & 1
\end{array}\right) \\
=\left(\begin{array}{ll}
1 & 0 \\
\beta & 1
\end{array}\right)\left(\begin{array}{cc}
A & 0 \\
-\beta A & D
\end{array}\right) \\
=\left(\begin{array}{cc}
A & 0 \\
-\beta D & D
\end{array}\right)\left(\begin{array}{ll}
1 & 0 \\
\beta & 1
\end{array}\right)
\end{aligned}
\end{aligned}
$$

II faut maintenant rechercher des réalisations pratiques de ces matrices $\alpha$ et $\beta$. La matrice $\beta$ peut être représentée comme une mise en cascade d'une lentille mince et d'un apodiseur gaussien (transmission en amplitude égale à exp(- $\left.a_{2} x^{2} / 2\right)$ ) qui s'écrit :

$$
\left(\begin{array}{cc}
1 & 0 \\
-\frac{1}{f} & 1
\end{array}\right)\left(\begin{array}{cc}
1 & 0 \\
-j \frac{\lambda_{0 a_{2}}}{2 \pi} & 1
\end{array}\right)=\left(\begin{array}{cc}
1 & 0 \\
-\frac{1}{f}-j \frac{\lambda_{0 a_{2}}}{2 \pi} & 1
\end{array}\right)=\left(\begin{array}{ll}
1 & 0 \\
\beta & 1
\end{array}\right), \beta \in C(11 . g)
$$

Si $\alpha \in \mathbb{R}^{+}$, la matrice $\alpha$ est simplement une matrice de propagation libre. Dans le cas général, $\alpha$ peut être décomposée en 3 lentilles de focale complexe (matrice $\beta$ ) séparées par 2 milieux uniformes.

$$
\begin{aligned}
\left(\begin{array}{ll}
1 & \alpha \\
0 & 1
\end{array}\right)= & \left(\begin{array}{cc}
1 & 0 \\
2\left(\alpha^{-1}-\mid-1\right) & 1
\end{array}\right) \cdot\left(\begin{array}{cc}
1 & 1 / 2 \\
0 & 1
\end{array}\right) \cdot\left(\begin{array}{cc}
1 & 0 \\
4\left(\left.\alpha\right|^{-2}-\left.\right|^{-1}\right) & 1
\end{array}\right) . \\
& \left(\begin{array}{cc}
1 & 1 / 2 \\
0 & 1
\end{array}\right) \cdot\left(\begin{array}{cc}
1 & 0 \\
2\left(\alpha^{-1}-\left.\right|^{-1}\right) & 1
\end{array}\right) \quad(11 . h)
\end{aligned}
$$

avec $\mathrm{I}$ = longueur du système qui est arbitraire et peut donc servir à ajuster l'encombrement d'un dispositif. 


\section{Exemple.}

Trouver un système optique dont le faisceau de sortie est identique au faisceau gaussien incident.

On cherche donc à décomposer la matrice identité.

$\| \mathrm{y}$ a une infinité de solutions à ce problème.

On peut par exemple écrire, d'après (11.d) :

$$
\left(\begin{array}{ll}
1 & 0 \\
0 & 1
\end{array}\right)=\left(\begin{array}{cc}
1 & -I_{1} \\
0 & 1
\end{array}\right)\left(\begin{array}{ll}
1 & I_{1} \\
0 & 1
\end{array}\right) \text { où lis est arbitraire }
$$

La première matrice étant décomposée selon le schéma (11.h), on aboutit à :

$\left(\begin{array}{ll}1 & 0 \\ 0 & 1\end{array}\right)=\left(\begin{array}{cc}1 & 0 \\ -2\left(I_{1}-^{-1}+I_{2}-1\right) & 1\end{array}\right) \cdot\left(\begin{array}{cc}1 & I_{2} / 2 \\ 0 & 1\end{array}\right) \cdot\left(\begin{array}{cc}1 & 0 \\ -4\left(I_{1} I_{2}-2+I_{2}-1\right) & 1\end{array}\right)$.

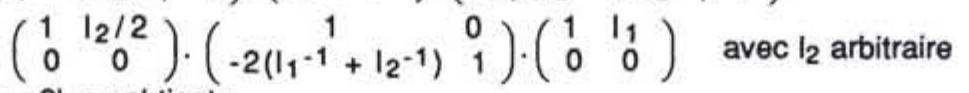

Si on pose $l_{2}=2 l_{1}=2 l$, on obtient :

$\left(\begin{array}{ll}1 & 0 \\ 0 & 1\end{array}\right)=\left(\begin{array}{cc}1 & 0 \\ -3 / / & 1\end{array}\right) \cdot\left(\begin{array}{ll}1 & 1 \\ 0 & 1\end{array}\right) \cdot\left(\begin{array}{cc}1 & 0 \\ -3 / 1 & 1\end{array}\right) \cdot\left(\begin{array}{ll}1 & 1 \\ 0 & 1\end{array}\right) \cdot\left(\begin{array}{cc}1 & 0 \\ -3 / 1 & 1\end{array}\right) \cdot\left(\begin{array}{ll}1 & 1 \\ 0 & 1\end{array}\right)$

qui est représenté en figure 9 .

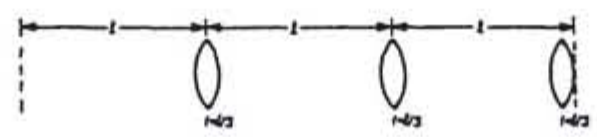

- Fig 9 -

\section{Les faisceaux réels [6]}

En pratique, le faisceau d'un laser n'est jamais purement gaussien (troncature, distorsion de phase,...). Dès qu'on souhaite extraire un maximum de puissance optique d'un milieu amplificateur, le faisceau est plutôt du type multimode transverse (cavité stable) ou est annulaire (cavité instable). La théorie de la propagation des faisceaux gaussiens exposée jusqu'à présent n'est plus valable.

Les premières difficultés de quantification d'un faisceau réel résident dans la détermination des dimensions transversales de la distribution d'intensité et dans la détermination de l'axe optique du faisceau comme l'illustre la figure 10.

Depuis peu, une théorie a émergé décrivant les caractéristiques d'un faisceau quelconque à partir des moments d'ordre un et deux de la distribution d'intensité. Ces moments peuvent être propagés au travers d'un système optique décrit par une matrice ABCD. 


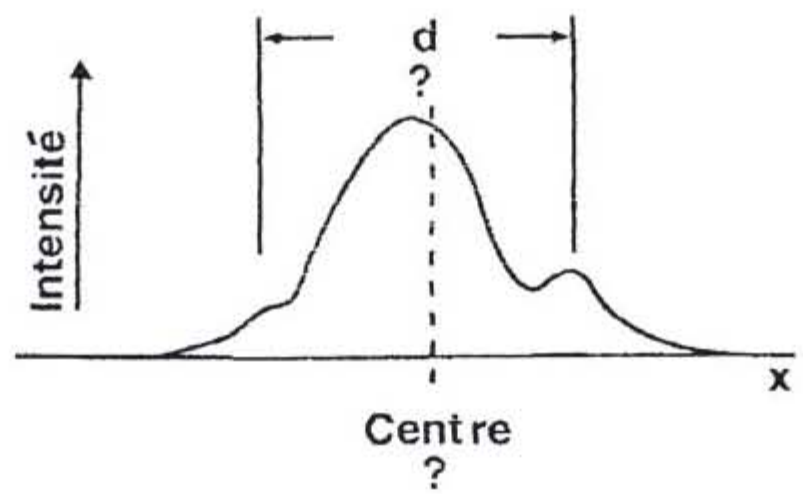

- Fig. 10 -

\subsection{Moment d'ordre 1 et propagation.}

Plaçons nous dans le cas unidimensionnel avec $u(x, z)$ tel que $\int_{-\infty}^{+\infty}|u|^{2} d x=1$.

Soit $P\left(s_{x}, z\right)$ la transformée de Fourier de $u(x, z)$.

$$
u(x, z)=\int_{-\infty}^{+\infty} P\left(s_{x}, z\right) \exp \left(-2 \pi j s_{x} x\right) d s_{x}
$$

On peut montrer [5], que dans le cadre de l'approximation paraxiale, $\mathrm{P}\left(\mathrm{s}_{\mathrm{x}}, \mathrm{z}\right)$ se propage sous la forme:

$$
P\left(s_{x}, z_{1}\right)=P\left(s_{x}, z_{0}\right) \exp \left[j \pi \lambda s_{x}^{2}\left(z_{1}-z_{0}\right)\right] \text { (12) }
$$

Considérons les 2 moments d'ordre 1 :

$$
\bar{x}=\int_{-\infty}^{+\infty} x|u(x, z)|^{2} d x \quad \text { et } \quad \overline{s_{x}}=\int_{-\infty}^{+\infty} s_{x}\left|P\left(s_{x}, z\right)\right|^{2} d s_{x}
$$

On montre en utilisant des théorèmes sur la transformation de Fourier que :

$$
\text { et } \begin{aligned}
\bar{x}\left(z_{1}\right) & =\bar{x}\left(z_{0}\right)+\lambda\left(z_{1}-z_{0}\right) \overline{s_{x}} \\
\text { et } \quad \bar{s}_{x}\left(z_{1}\right) & =\bar{s}_{x}\left(z_{0}\right)=\text { constante. }
\end{aligned}
$$

Par conséquent, le barycentre de la distribution d'intensité se propage suivant une droite (dans le cadre de l'approximation paraxiale) et il est alors naturel de prendre cette droite comme étant l'axe optique du faisceau.

\subsection{Mement d'ordre 2 et propagation.}

On définit 2 moments d'ordre 2 : 


$$
\begin{aligned}
& \sigma_{x}^{2}(z)=\int_{-\infty}^{+\infty}(x-\bar{x})^{2}|u(x, z)|^{2} d x \\
& \sigma_{s x}{ }^{2}(z)=\int_{-\infty}^{+\infty}\left(s_{x}-\overline{s_{x}}\right)^{2}\left|P\left(s_{x}, z\right)\right|^{2} d s_{x}
\end{aligned}
$$

On montre que $\sigma_{x}{ }^{2}(z)$ présente un minimum $\sigma_{x}^{2}\left(z_{0}\right)=\sigma_{x_{0}}^{2}$ situé en $z=z_{0 x}$

tel que $\quad \sigma_{x}^{2}(z)=\sigma_{x_{0}}^{2}+\lambda^{2}\left(z-z_{0 x}\right)^{2} \sigma_{S_{x}}^{2}$

et

$$
\sigma_{S_{x}}^{2}=\sigma_{S_{x_{0}}}^{2}=\text { constante }
$$

\subsection{Facteur de qualité}

$u(x, z)$ et $P\left(s_{x}, z\right)$ étant liés par une transformation de Fourier, on peut écrire la relation d'incertitude :

$$
\sigma_{\mathrm{x}} \cdot \sigma_{\mathrm{S}} \geq \frac{1}{4 \pi}
$$

où l'égalité a lieu lorsque $u$ est une gaussienne.

Pour le faisceau réel ( $\left.\sigma_{\mathrm{x}} \cdot \sigma_{\mathrm{S}_{\mathrm{x}}}>\frac{1}{4 \pi}\right)$, on introduit un nombre sans dimension $M_{x}{ }^{2} \geq 1$ appelé "facteur de qualité" tel que :

$$
\sigma_{x_{0}} \cdot \sigma_{\mathrm{S}_{0}}=\frac{M_{x}^{2}}{4 \pi}
$$

Si on définit le rayon du faisceau réel $W_{x}(z)$ par analogie avec le faisceau gaussien TEM 00 alors $W_{x}(z)=2 \sigma_{x}(z)$ et $\quad \sigma_{S_{x}}=\frac{1}{2 \pi W_{0 x}}$

On peut réécrire (15) de la façon suivante :

$$
W_{x}^{2}=W_{0 x^{2}}\left(1+\left(\frac{z-z_{0 x}}{\left(\frac{\pi W_{0 x}^{2}}{M_{x}^{2} \lambda}\right)}\right)^{2}\right)
$$

Le paramètre de Rayleigh et la demi-divergence sont alors

$$
Z_{R_{x}}=\frac{\pi W_{0 x}{ }^{2}}{M_{x}{ }^{2} \lambda} \quad \text { et } \quad \theta_{1 / 2}=\frac{M_{x}{ }^{2} \lambda}{\pi W_{0 x}}
$$

La relation (15. bis) se réécrit sous la forme :

$$
W_{x}^{2}=W_{0 x^{2}}\left(1+\left(\frac{z-z_{0 x}}{Z_{R_{x}}}\right)^{2}\right)
$$

Toutes ces équations et définitions sont à rapprocher des formules de propagation libre établies pour le faisceau gaussien TEM 00 ; la seule différence consiste dans l'introduction du facteur de qualité $\mathrm{M}^{2}$; dans toutes les expressions établies pour les faisceaux gaussiens $\lambda$ est changé en $M^{2} \lambda$. 


\subsection{Propagation d'un faisceau réel au travers d'un système optique paraxial.}

Considérons un système optique paraxial décrit par une matrice en $A B C D$. On peut montrer [7] que le facteur de qualité $M_{x}{ }^{2}$ est un invariant et qu'on peut définir un rayon complexe $Q_{x}$ pour le faisceau réel qui se transforme suivant l'expression

$$
Q_{x_{2}}=\frac{A Q_{x_{1}}+B}{C Q_{x_{1}}+D} \quad \text { (18) } \quad \text { avec } \frac{1}{Q_{x}}=\frac{1}{R_{x}(z)}-j \frac{M_{x}^{2} \lambda}{\pi W_{x}^{2}(z)}
$$

où $R_{x}(z)$ est un rayon de courbure moyen du faisceau réel définit par :

$$
\frac{1}{R_{x}(z)}=\frac{1}{j 4 \pi \sigma_{x^{2}}} \int_{-\infty}^{+\infty} x\left(u^{*} \frac{d u}{d x}-u^{\frac{d u^{*}}{d x}}\right) d x
$$

Le résultat (18) est en tout point identique à la relation de propagation du rayon complexe pour un faisceau gaussien TEMoo.

II faut noter que toutes les équations de ce chapitre sont valables pour une propagation au travers d'un système optique limité par la diffraction. Tout élément aberrant ou toute diaphragmation modifie les caractéristiques du faisceau (en aprticulier $\mathrm{M}_{\mathrm{x}}{ }^{2}$ ) ; dans ces cas, la mesure (ou le calcul par code numérique) des paramètres réels du faisceau après l'élément perturbateur est necessaire pour pouvoir reprendre le calcul de propagation suivant la méthode exposée ci-dessus.

\section{Transpert des faisceaux laser de puissance.}

Jusqu'à présent, on a décrit la propagation des faisceaux laser comme un phénomène linéaire où le milieu de propagation est supposé ne pas être affecté par le faisceau laser. Ceci n'est vrai que pour des faisceaux de faible intensité. Dès que l'intensité devient importante, les propriétés optiques du milieu de propagation sont altérées. Ce phénomène est non linéaire dans le sens où la distribution d'intensité génère un changement d'indice de réfraction qui à son tour altère la distribution d'intensité, qui altère l'indice de réfraction et ainsi de suite...

Dans le cadre de ce cours, on exposera quelques phénomènes thermiques liés à la propagation et ceci de manière qualitative.

\subsection{Epanouissement Laser : ("Thermal Blooming") [8]}

Les longueurs d'onde laser, bien que choisies dans les "fenêtres" de transmission de l'atmosphère, subissent des absorptions résiduelles par les molécules et les aérosols présents dans l'atmosphère.

Considérons un faisceau gaussien se propageant dans une atmosphère stationnaire (température $T_{0}$, densité $\rho_{0}$, indice $n_{0}$ ). L'absorption résiduelle des photons par les molécules et les aérosols conduit à un accroissement de la température sur le parcours du faisceau (Fig. 11a).

L'air, plus chaud au centre, se dilate radialement et cause une chute de la densité donc de l'indice de l'air au voisinage de l'axe du faisceau. Les rayons proches de l'axe voient un gradient d'indice positif important et sont défléchis vers l'extérieur du faisceau donnant naissance à un nouveau profil d'intensité.

Dans le cas d'un vent latéral (Fig. 11b), on obtient un résultat un peu similaire.

Considérons une cellule d'air traversant la distribution d'intensité du faisceau laser. Au fur et à mesure de son déplacement, la température de cette cellule augmente pour être maximale en sortie de la section transverse du faisceau. L'indice de réfraction est plus faible en aval et 
258 A. Culoma
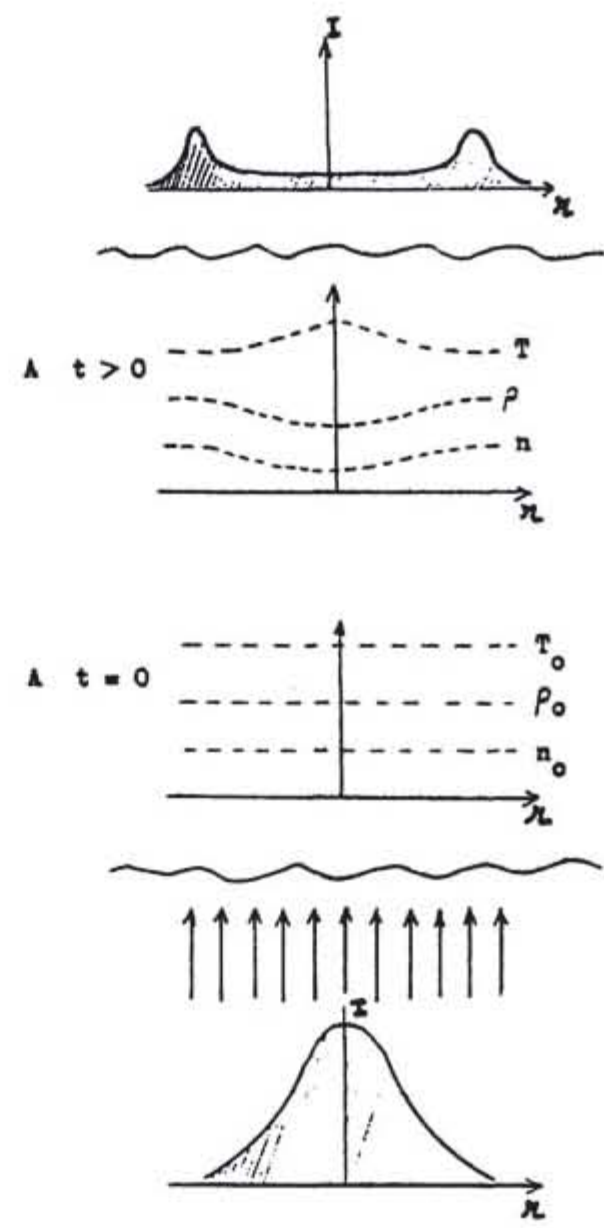

Fif $11-a$ 

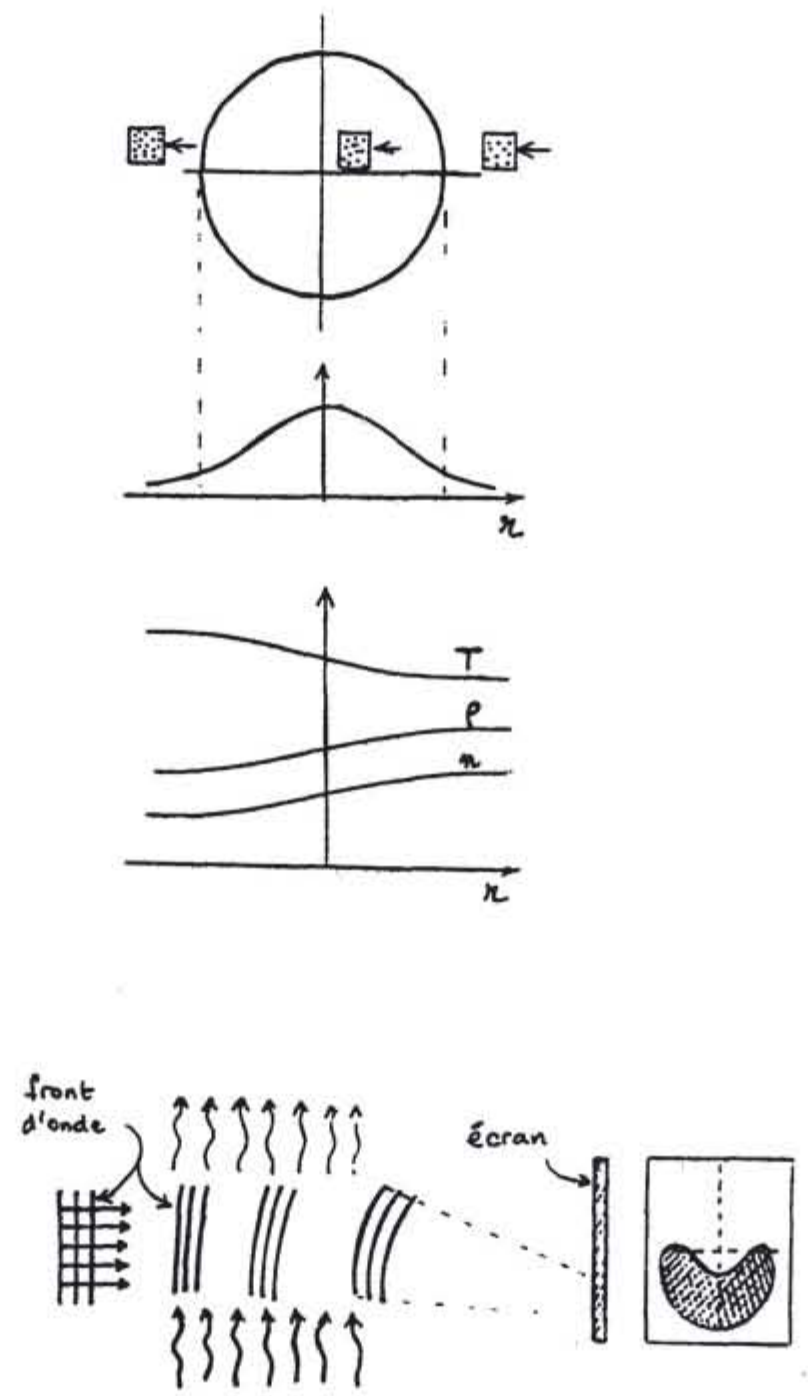

-Fig 11.6 - 
les rayons sont défléchis vers l'amont du vent transverse. La distribution d'intensité est distordue et exhibe une forme caractéristique en croissant.

\section{2 "Lentille thermique" dans les solides [9]}

Considérons une lame à faces planes et parallèles de faible épaisseur b $<$ D, soumise à la traversée d'un faisceau laser. Dû à l'absorption résiduelle du substrat et des éventuelles couches minces optiques sur les faces d'entrée et de sortie, un gradient de température va se développer dans le substrat qui va avoir tendance à "épaissir" le centre de la lame. Ce gradient de température va également causer un gradient d'indice dans la lame puisque l'indice est fonction de la température.

Les rayons lumineux incidents vont être déviés par la distorsion mécanique des interfaces et par le gradient dindice induit. (Fig. 12).

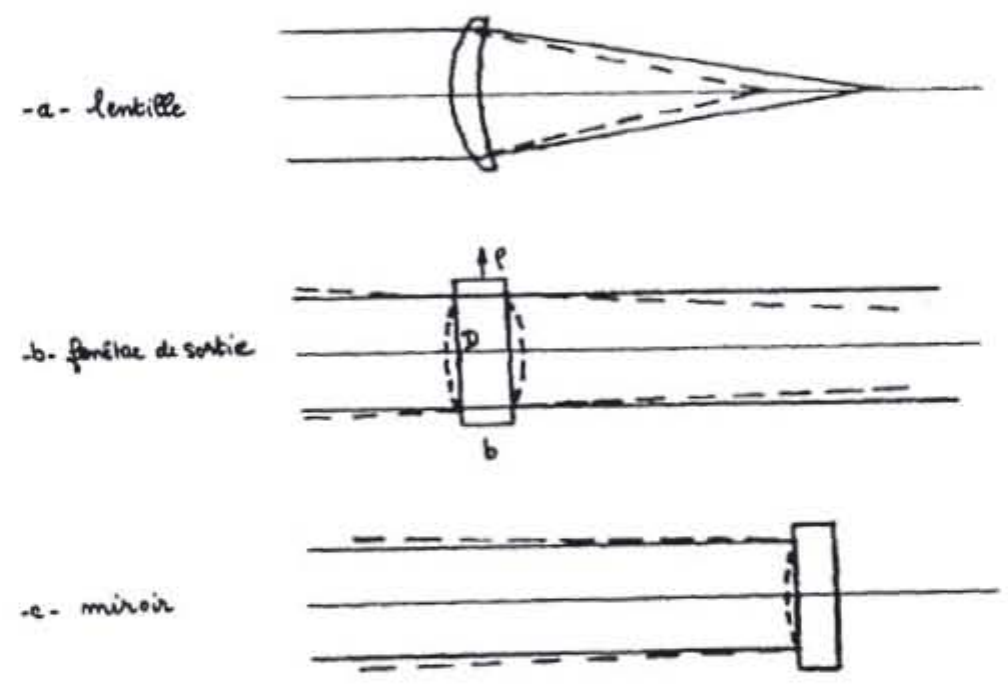

- Fig. 12. -

Dans le cas où le gradient d'indice est de forme parabolique

$$
T(\rho)=T(0) \cdot\left(\frac{2 \rho}{D}\right)^{2} \Delta T
$$

et les contraintes mécaniques négligées, la focale thermique induite est de la forme :

$$
\frac{1}{F_{t h}}=8\left(\left(\frac{\partial n}{\partial T}\right)_{\sigma}=0+\alpha(1+v)(n-1)\right) \frac{b}{D^{2}} \Delta T
$$

avec $\quad \alpha=$ coefficient de dilatation linéaire

$v=$ coefficient de Poisson 


\subsection{Choix des composants optiques - Facteur de mérite [10]}

On peut définir pour les matériaux de faible absorption pouvant être utilisés dans le transport des faisceaux laser de puissance, une figure de mérite qui dans le cas d'un hublot regroupe tous les termes ayant trait à la génération de la focalisation thermique :

$$
\begin{gathered}
F_{O M_{H}}=\frac{c_{D}^{\prime}}{\left(2 \beta_{S}+\beta_{v} b\right) \chi} \\
\text { où } x=(n-1) \alpha(1+v)+\left(\frac{\partial n}{\partial T}\right)_{\sigma=0}+\frac{n^{3} \alpha E}{4}\left(B_{/ /}+B_{\perp}\right)
\end{gathered}
$$

avec $E=$ module $d^{\prime}$ Young

$B_{/ /}$et $B_{\perp}=$ coefficient élasto-optique

$\beta_{\mathrm{S}}=$ absorption de surface

$\beta_{v}=$ absorption de volume par unité de longueur

$c_{p}^{\prime}=$ capacité calorifique

On peut de même définir un facteur de mérite pour un miroir refroidi sous la peau : (Fig. 13)

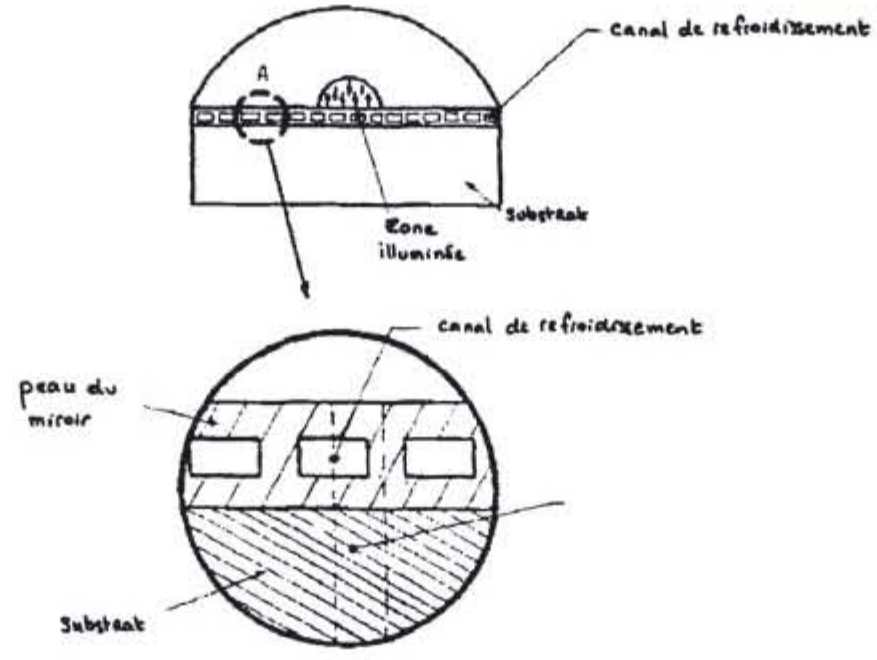

- Fig. 13 -

$$
F_{O M_{M}}=\frac{K}{\left.(1-R)\right|^{2} \alpha(1+v)\left(1+\frac{2}{N u}\right)}
$$

avec $\mathrm{K}=$ conductivité thermique

$(1-\mathrm{R})=$ coefficient d'absorption du miroir

1 = épaisseur de peau

$\mathrm{Nu}=$ nombre de Nusselt caractérisant le refroidissement.

La figure 14 extraite de la référence [10] donne quelques exemples de figures de mérite pour des matériaux classiquement utilisés. 


\section{Conclusion.}

Ce cours présente les aspects les plus utiles dans l'étude des caractéristiques dimensionnelles d'un système optique réalisé à l'aide d'un laser. La propagation spatiale "globale des faisceaux réels est traitée de façon rigoureuse à partir des moments d'ordre 1 et 2 de la distribution d'intensité.

Le transport des faisceaux de puissance est traité d'un point de vue thermique conduisant à des figures de mérite déterminant le choix des matériaux.

\section{Bibliographie.}

[1]. P. BAUES Opto-Electronics 1, p.37-44 (1969)

[2]. W.H. STEIER Applied Optics 5, p. 1229

3]. P. BELLAND : J.P. CRENN Applied Optics 21, p. 522-527 (1982)

[4]. L.W. CASPERSON Applied Optics 20, p. 2243-2249 (1981)

15. J.W. GOODMAN Introduction to Fourier Optics (Mc. Graw. Hill 1968)

[6]. A.E. SIEGMAN New developments in Laser Resonators SPIE 1224 (1990)

[7]. P.A. BELANGER Optics Letters 16, p. 196-198 (1991)

18. F.G. GEBHARDT Applied Optics 15, p. 1479 (1976)

[9]. M. SPARKS Journal of Applied Physics 42, p. 5029-5046 (1971)

[10]. C.A. KLEIN Optical Engineering 18, p. 591-601

(1979)

[11]. ouvrage général traitant des faisceaux laser : A.E. SIEGMAN LASERS University Science Books 